\title{
Template-directed assembly of short peptides into layered porous frameworks
}

\author{
Dmitriy V Soldatov, Aaron J Smith \\ Department of Chemistry, University of Guelph, Canada \\ E-mail: soldatov@uoguelph.ca
}

In comparison with their long-chain analogs, short peptides display a much greater diversity of their $\mathrm{H}$-bonded frameworks in the crystals. Moreover, the same peptide can be assembled in several topologically different frameworks when co-crystallized with other molecules acting as templates. When the $\mathrm{H}$-bonding motifs can be envisioned, the templating approach becomes a powerful tool of generating porous frameworks in a rational way, thus creating new materials with a desired structure and properties.

Our recent studies explored the template-directed assembly of dipeptides and tripeptides into layered porous frameworks within the isolated co-crystals and inclusion compounds. The peptides with bulky hydrophobic side groups (leucyl, isoleucyl, valyl) were used to expand the structure and facilitate inclusion of template species into the interlayer space. Two or more $\mathrm{H}$ bonded frameworks were consistently generated using this approach, with parallel, antiparallel and crisscross arrangement of the molecules in the peptide layer. Once created, the peptide framework is both stable and remarkably flexible, undergoing contraction/expansion or more sophisticated transformations without loosing its $\mathrm{H}$-bond connectivity. The changes induced by temperature, template exchange and guest removal have been studied. The crystal structures were often complicated with twinning, extensive disorder, and partial occupancy of additionally included solvent molecules.

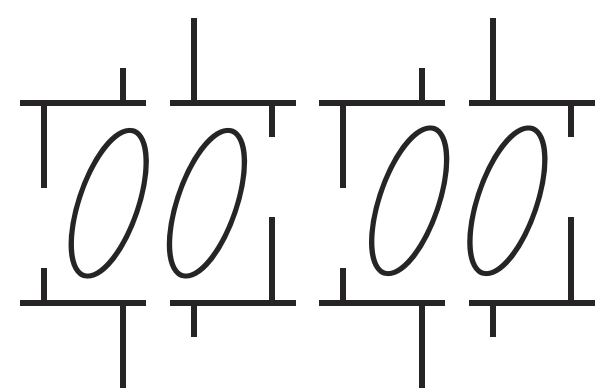

Schematic view of template species filling the space between two peptide layers 